\title{
ABSTRACT FUNCTIONS WITH CONTINUOUS DIFFERENCES AND NAMIOKA SPACES
}

\author{
BOLIS BASIT AND HANS GÜNZLER
}

\begin{abstract}
Let $G$ be a semigroup and a topological space. Let $X$ be an Abelian topological group. The right differences $\triangle_{h} \varphi$ of a function $\varphi: G \rightarrow X$ are defined by $\triangle_{h} \varphi(t)=\varphi(t h)-\varphi(t)$ for $h, t \in G$. Let $\triangle_{h} \varphi$ be continuous at the identity $e$ of $G$ for all $h$ in a neighbourhood $U$ of $e$. We give conditions on $X$ or range $\varphi$ under which $\varphi$ is continuous for any topological space $G$. We also seek conditions on $G$ under which we conclude that $\varphi$ is continuous at $e$ for arbitrary $X$. This led us to introduce new classes of semigroups containing all complete metric and locally countably compact quasitopological groups. In this paper we study these classes and explore their relation with Namioka spaces.
\end{abstract}

\section{INTRODUCTION, NOTATION AND MAIN DEFINITIONS}

Unless otherwise specified $G$ will stand for a topological space (usually (semi) group) and $X$ for an Abelian Hausdorff topological group with identity 0 . If $X$ is a locally convex space and $p$ is one of its continuous seminorms, we set $X_{o}=\{x \in$ $X: p(x)=0\}$. It follows that $X_{p}=X / X_{o}$ is a normed space. We denote by $j$ the natural homomorphism of $X$ onto $X / X_{o}$. Let $C(G, G)$ be the space of continuous functions from $G$ to $G$. We assume that $\varphi: G \rightarrow X$ satisfies the following property at the point $t_{o}$ of the open set $U \subset G$ :

(i) for each $t \in U$ there exists $h \in C(G, G)$ such that $h\left(t_{o}\right)=t$,

(ii) $T_{h} \varphi=\varphi \circ h-\varphi$ is continuous at $t_{o}$.

If $G$ is a semigroup and $\varphi: G \rightarrow X$, then $\Delta_{h} \varphi(t):=\varphi(t h)-\varphi(t)$ and $\Delta^{h} \varphi(t):=$ $\varphi(h t)-\varphi(t)$ will denote respectively the right and the left difference by $h ; \rho_{h}, \lambda_{h}$ will stand for the mappings defined by $\rho_{h}(t)=t h, \lambda_{h}(t)=h t$ for all $h, t \in G$.

This paper is concerned with the following problems

(P.1) Find conditions on $X$ or range $\varphi$ under which $\varphi$ satisfying (tr-d) is continuous at $t_{o}$ for arbitrary space $G$.

(P.2) Find conditions on $G$ under which $\varphi$ satisfying (tr-d) is continuous at $t_{o}$ for each locally convex space $X$.

We give a necessary and sufficient condition to solve (P.1) and obtain new results for differences on semigroups. We investigate (P.2) in the case: $G$ is a semigroup

Received by the editors May 4, 1995.

1991 Mathematics Subject Classification. Primary 28B05, 39A05; Secondary 90D05, 54C05, $54 \mathrm{E} 35$.

Key words and phrases. Differences, weak continuity, Namioka spaces, right uniform continuity, Baire spaces, Banach spaces not containing $c_{0}$.

(C)1996 American Mathematical Society 
with identity $e, X$ is a locally convex linear space and condition (tr-d) is replaced by

$$
\text { for each } h \in U \text {, the right difference } \triangle_{h} \varphi \text { is continuous }
$$
at $e$.

The study of (P.2) led us to introduce the following classes:

Definition 1.1. Let $G$ be a semigroup with identity $e$ and a topology. Then

(i) $G$ is called of D-type if and only if there exists a sequence $\left(V_{n}\right)$ of neighbourhoods of $e$ such that

$$
\begin{aligned}
& \text { for any sequence }\left(t_{n}\right) \text { with } t_{n} \in V_{n} \text { for } n \in \mathbf{N} \text {, the } \\
& \text { sequence }\left(\tau_{m}\right) \text { defined by } \tau_{m}:=t_{m} t_{m-1} \ldots t_{1} \text { for } m \in \mathbf{N} \text {, } \\
& \text { has a convergent subnet. }
\end{aligned}
$$

(ii) $G$ is called of $\Delta$-type [respectively weak $\Delta$-type] if and only if for each neighbourhood $U$ of $e$ and each function $\varphi: G \rightarrow X$ with $X$ a locally convex space, $\varphi$ bounded on $U$ and $\triangle_{h} \varphi$ continuous at $e$ for all $h \in U$ [ respectively all $\left.h \in G\right], \varphi$ is continuous at $e$.

Definition 1.2. A Hausdorff topological space $G$ is called a weak Namioka space if and only if for each compact $Y$, each separately continuous function $\Phi: G \times Y \rightarrow \mathbf{R}$ and each nonvoid open $U \subset G$, there exists $a \in U$ such that $\Phi$ is continuous on $\{a\} \times Y$.

Similar concepts with game-theoretic interpretations have been introduced by Choquet [7, p. 106], Christensen [8] and Saint-Raymond [15]. We note that Namioka spaces (see [15]) are weak Namioka spaces.

Finally, for the convenience of the reader, we recall the following definitions.

Let $G$ be a semigroup and a topological space (see [4, p. 26]). Then $G$ is called

$(\alpha)$ a right (left) semitopological semigroup if the map $\rho_{h}\left(\lambda_{h}\right)$ is continuous for all $h \in G$;

$(\beta)$ a semitopological semigroup if the maps $\rho_{h}$ and $\lambda_{h}$ are continuous for all $h \in G$;

$(\gamma)$ a topological semigroup if the multiplication $(s, t) \rightarrow s t$ from $G \times G$ to $G$ is continuous.

$(\delta)$ a semitopological (quasitopological) group if $G$ is a group and a semitopological (topological) semigroup.

Let $G$ be a semitopological group, $X$ be a Hausdorff Abelian group and $\varphi: G \rightarrow X$. Then $\varphi$ is right (left) uniformly continuous if for each neighbourhood $W$ of 0 of $X$ there exists a neighbourhood $V$ of $e$ such that $\Delta_{v} \varphi(t) \in W\left(\Delta^{v} \varphi(t) \in W\right)$ for all $v \in$ $V$ and $t \in G . \varphi$ is uniformly continuous if it is left and right uniformly continuous. If $G$ is a totally bounded quasitopological group, then $\varphi$ is right uniformly continuous if and only if it is left uniformly continuous.

This paper consists of five sections. In section 2 we show that the conditions given in [2] in the case $(\Delta)$ are also necessary and sufficient to solve (P.1) for any topological space $G$ and $\varphi$ with (tr-d). We also extend and improve recent results in [16]. In section 3 , we prove that D-type quasitopological regular groups are $\sigma$ complete Baire spaces (Theorem 3.2) and a weak Namioka semitopological group is of weak $\Delta$-type (Proposition 3.4). The main result of section 4 states: D-type quasitopological regular groups are of $\Delta$-type. In section 5 , we refine and extend several results on uniformly continuous differences $[3,9,10,11]$. 


\section{DIFFERENCES ON TOPOLOGICAL SPACES}

In this section we study continuity of functions defined on a topological space $G$ with values in an Abelian Hausdorff topological group $X$. We assume that $\varphi: G \rightarrow X$ satisfies property (tr-d) (see the introduction) at the point $t_{o}$ of the open subset $U \subset G$. Special $G$ satisfying (tr-d) (i) are semitopological groups or homogeneous topological spaces.

If $X$ is a linear topological space, we write $c_{o} \not \subset X$ if and only if $X$ does not contain a subspace isomorphic to $c_{o}$ (the Banach space of real valued sequences convergent to 0$)$. We call the subset $\varphi(U)$ of $X$ relatively weakly sequentially complete if and only if every weak Cauchy sequence from it converges weakly to an element from $X$. The spaces $X=\mathbf{C}^{n}$ for some $n \in \mathbf{N}$ and $X$ weakly sequentially complete Banach spaces satisfy $c_{o} \not \subset X$.

Theorem 2.1. Let $\{\varphi, U, X\}$ satisfy one of the following

(a) $X$ is a Fréchet space (i.e. complete locally convex metric) and $c_{o} \not \subset X$.

(b) $X$ is a sequentially complete locally convex space and $\varphi(U)$ is relatively weakly sequentially complete.

Let $\varphi: G \rightarrow X$ with arbitrary topological space $G$ be bounded on $U$ and satisfy (tr-d). Then $\varphi$ is continuous at $t_{o}$.

Proof. First, we prove the case where $X$ is a Banach space. Let $\varphi\left(t_{o}\right)=0$. Assuming the contrary, there exists $\epsilon_{o}>0$, a sequence $\left(t_{n}\right) \subset U$ and a net $\left(h_{i}\right)_{i \in F} \subset C(G, G)$, where $F$ is the set of all finite subsets $i=\left\{n_{1}, n_{2}, \ldots, n_{m}\right\}$ of $\mathbf{N}$ such that $n_{1}<n_{2}<\ldots<n_{m}$, satisfying the following :

$$
\begin{gathered}
\left\|\varphi\left(t_{n}\right)\right\| \geq \epsilon_{o} \quad \text { and } \quad t_{n} \in U \text { for all } n \in \mathbf{N} ; \\
h_{n, n_{m}, n_{m-1}, \ldots, n_{1}}\left(t_{o}\right)=h_{n_{m}, n_{m-1}, \ldots, n_{1}}\left(t_{n}\right) \in U, \quad h_{n}\left(t_{o}\right)=t_{n}
\end{gathered}
$$

for all $1 \leq n_{1}<\ldots<n_{m}<n, m \in \mathbf{N}$; and $\varphi-\varphi \circ h_{i}$ is continuous at $t_{o}$, for all $i \in F$.

$$
\begin{array}{r}
\left\|\varphi\left(h_{n, n_{m}, n_{m-1}, \ldots ., n_{1}}\left(t_{o}\right)\right)-\varphi\left(t_{n}\right) \varphi\left(h_{n_{m}, n_{m-1}, \ldots, n_{1}}\left(t_{o}\right)\right)\right\|<\epsilon_{o} / 2^{n} \\
\text { for all } 1 \leq n_{1}<\ldots<n_{m}<n, n, m \in \mathbf{N} .
\end{array}
$$

Indeed, denote by $i(n)=\{j \in F: j \subset\{1,2, \ldots, n\}\}, U_{n}=\left\{t: h_{i}(t) \in U\right.$, for all $i \in$ $i(n)\} \cap U$ and $W_{n}(\epsilon)=\left\{t:\left\|\Delta_{i} \varphi(t)-\Delta_{i} \varphi\left(t_{o}\right)\right\|<\epsilon\right.$, for all $\left.i \in i(n)\right\} \cap U$. By assumptions, there exists $\epsilon_{o}>0$ and $t_{1} \in U$ such that $\left\|\varphi\left(t_{1}\right)\right\| \geq \epsilon_{o}$. Choose $h_{1} \in C(G, G)$ such that $h_{1}\left(t_{o}\right)=t_{1}$ and $\varphi-\varphi \circ h_{1}$ is continuous at $t_{o}$. We proceed by induction. If $\left\{t_{1}, t_{2}, \ldots, t_{n-1}\right\}$ and $\left\{h_{i}: i \in i(n-1)\right\}$ are chosen satisfying (2.1)(2.3), we select $t_{n} \in U_{n-1} \cap W_{n-1}\left(\epsilon_{o} / 2^{n-1}\right)$ such that $\left\|\varphi\left(t_{n}\right)\right\| \geq \epsilon_{o}$. Then we choose $h_{n, n_{m}, n_{m-1}, \ldots, n_{1}}$ satisfying (2.2). Clearly (2.1)-(2.3) are satisfied. Let $\pi=\left(n_{k}\right)$ with $n_{k}<n_{k+1}$ for all $k \in \mathbf{N}$. Set $s_{m}=\sum_{k=1}^{m} \varphi\left(t_{n_{k}}\right)$ and $\tilde{s}_{m}=\varphi\left(h_{n_{m}, n_{m-1} \ldots, n_{1}}\left(t_{o}\right)\right)$. Let $\sigma_{m}=s_{m}-\tilde{s}_{m}$. From the identity

$$
\begin{aligned}
\sigma_{m}-\sigma_{m+p}= & \varphi\left(h_{n_{m+p}, \ldots, n_{1}}\left(t_{o}\right)\right)-\varphi\left(t_{n_{m+p}}\right)-\varphi\left(h_{n_{m+p-1}, \ldots, n_{1}}\left(t_{o}\right)\right) \\
& +\ldots .+\varphi\left(h_{n_{m+1}, \ldots, n_{1}}\left(t_{o}\right)\right)-\varphi\left(t_{n_{m+1}}\right)-\varphi\left(h_{n_{m}, \ldots, n_{1}}\left(t_{o}\right)\right),
\end{aligned}
$$

and $(2.3)$, we conclude that $\left(\sigma_{m}\right)$ is a Cauchy sequence. Denote its limit by $y_{\pi}$. We show that

$$
\left(s_{m}\right) \text { and }(\tilde{s})_{m} \text { are weakly sequentially Cauchy. }
$$


Indeed, from the identity $s_{m}=\sigma_{m}+\tilde{s}_{m}$, we get $\left(s_{m}\right)$ is bounded. This means that for each subseries $\sum_{k=1}^{\infty} \varphi\left(t_{n_{k}}\right)$ the sequence $\left(s_{m}\right)$ of partial sums is bounded and hence $\sum_{n=1}^{\infty} \varphi\left(t_{n}\right)$ is weakly unconditionally convergent (see [5]). This implies that the sequence $\tilde{s}_{m}:=\varphi\left(h_{n_{m}, n_{m-1}, \ldots, n_{1}}\left(t_{o}\right)\right)$ is weakly sequentially Cauchy and under the condition (b), $\tilde{s}_{m} \rightarrow x_{\pi} \in X$ and $s_{m} \rightarrow y_{\pi}+x_{\pi}$ weakly as $m \rightarrow \infty$. Therefore under (a) or (b), by [5] or Orlicz's Theorem $\sum_{n=1}^{\infty} \varphi\left(t_{n}\right)$ is convergent, contradicting (2.1). The case $X$ is a Fréchet space can be treated similarly. To prove (b) when $X$ is locally convex we apply the above arguments to $j \circ \varphi$. This proves that $\varphi$ is continuous at $t_{o}$.

Corollary 2.2. Let $G$ be a right semitopological semigroup with identity e. Let $\varphi$ : $G \rightarrow X$ be bounded on some open neighbourhood $U$ of e and let $\Delta_{h} \varphi$ be continuous at e for all $h \in U$ [respectively all $h \in G$ ]. If either (a) or (b) of Theorem 2.1 holds, then $\varphi$ is continuous at $e$. If in addition,

(i) $V t$ is a neighbourhood of $t \in G$ if $V$ is for $e$, then $\varphi$ is continuous on $U$ [respectively on $G$ ]. In particular, if $G$ is a group, $\varphi$ is continuous on $U$ [respectively on $G]$.

(ii) $G$ is a totally bounded quasitopological group and $\Delta_{h} \varphi$ is uniformly continuous for all $h \in G, \varphi$ is uniformly continuous on $G$.

Proof. Since $G$ is a right semitopological semigroup with identity $e, \rho_{h} \in C(G, G)$ and $\rho_{h}(e)=h$ for all $h \in G$. We have $\varphi \circ \rho_{h}-\varphi=\Delta_{h} \varphi$ is continuous at $e$ for all $h \in U$. By Theorem 2.1, $\varphi$ is continuous at $e$. Using the additional condition (i), and the continuity of $\Delta_{h} \varphi$ at $e, \varphi$ is continuous at each point $h \in U$ [respectively $h \in G$. If $G$ is a group, the proof is obvious.

(ii) By (i), $\varphi$ is continuous on $G$. Let $E$ be a neighbourhood of $0 \in X$. Choose such a neighbourhood $E_{1}$ of 0 that $E_{1}+E_{1}-E_{1} \subset E$. There exists a neighbourhood $V$ of $e$ such that $\Delta_{v} \varphi(e) \in E_{1}$ for all $v \in V$. Since $G$ is a quasitopological group, there exists such a neighbourhood $V_{1}$ of $e$ that $V_{1} V_{1} \subset V$. Since $G$ is totally bounded, one can choose $\left\{t_{1}, \ldots, t_{n}\right\} \subset G$ such that $G=\bigcup_{k=1}^{n} V_{1} t_{k}$. Set $V_{2}=$ $\bigcap_{k=1}^{n}\left\{w:\left[\Delta_{t_{k}} \varphi(w t)-\Delta_{t_{k}} \varphi(t)\right] \in E_{1}\right.$ for all $\left.t \in G\right\} \cap V_{1}$. Let $t=\tau t_{k}$ for some $\tau \in V_{1}$ and $1 \leq k \leq n$. The identity

$$
\begin{aligned}
\varphi(v t)-\varphi(t) & =\varphi\left(v \tau t_{k}\right)-\varphi\left(\tau t_{k}\right) \\
& =\left[\Delta_{t_{k}} \varphi(v \tau)-\Delta_{t_{k}} \varphi(\tau)\right]+[\varphi(v \tau)-\varphi(e)]-[\varphi(\tau)-\varphi(e)]
\end{aligned}
$$

shows that $\Delta_{v} \varphi(t) \in E$ for all $v \in V_{2}$ and $t \in G$, proving that $\varphi$ is left uniformly continuous. Hence it is right uniformly continuous, by a statement above.

Let $G$ be a locally compact group. Recall that a function $\varphi: G \rightarrow X$ is weakly Haar measurable if and only if $x^{*} \circ \varphi$ is Haar measurable for all $x^{*} \in X^{*}$. A weakly Haar measurable function $\varphi$ is called continuous if and only if there is a continuous function $\psi: G \rightarrow X$ such that $\varphi=\psi$ almost everywhere on $G$. Denote by $L_{w}^{\infty}(G, X)$ the space of all bounded weakly measurable functions.

Corollary 2.3. Let $\Gamma$ be a dense subgroup of a compact topological group $G$ and let $X$ be a complete locally convex space. Let $\varphi: G \rightarrow X$ satisfy one of the following conditions:

(i) $\varphi$ is bounded and $\Delta_{h} \varphi \in C(G, X)$ for all $h \in \Gamma$.

(ii) $\varphi \in L_{w}^{\infty}(G, X)$, the dual space of $X$ has a countable separating set $M$ and $\Delta_{h} \varphi$ is continuous (in the above sense) for all $h \in \Gamma$. If either (a) or (b) of Theorem 2.1 holds, then 
if (i), the restriction $\left.\varphi\right|_{\Gamma}$ of $\varphi$ to $\Gamma$ is uniformly continuous and has a unique continuous extension $\Phi \in C(G, X)$. Moreover, $\psi(h):=\Delta_{h} \varphi$ is uniformly continuous on $\Gamma$.

if (ii), $\varphi$ is continuous in the above sense.

Proof. (i) Since $\Gamma$ is a dense subgroup of the compact group $G$, it is a totally bounded topological group. By Corollary 2.2 (ii), $\left.\varphi\right|_{\Gamma}$ is uniformly continuous. This implies that the range of $\left.\varphi\right|_{\Gamma}$ is a relatively compact subset of $X$. Since $X$ is complete, there exists a unique extension of $\left.\varphi\right|_{\Gamma}$ to a function $\Phi \in C(G, X)$. It follows that $\Psi(h):=\Delta_{h} \Phi, h \in G$, is a continuous function on $G$. It is easy to verify that $\psi(h)=\Delta_{h} \Phi, h \in \Gamma$. Hence $\psi=\left.\Psi\right|_{\Gamma}$ is a uniformly continuous function.

(ii) Denote by $\Psi_{h}$ the continuous representative of $\Delta_{h} \varphi$. We have $\Psi_{h k}(t)=$ $\Psi_{k}(t h)+\Psi_{h}(t)$ for all $h, k \in \Gamma$ and all $t \in G$. Define $\psi: \Gamma \rightarrow X$ by $\psi(h)=\Psi_{h}(e)$ for all $h \in \Gamma$. Then $\psi$ is a bounded function satisfying $\Delta_{h} \psi=\left.\Psi_{h}\right|_{\Gamma}$. By (i) $\psi$ is uniformly continuous on $\Gamma$ and has a unique continuous extension $\Psi \in C(G, X)$. Moreover, $\Psi(t)-\varphi(t)=\Psi(t h)-\varphi(t h)$ almost everywhere on $G$ for each fixed $h \in \Gamma$. Since $\Gamma$ is dense in $G$ and $\varphi$ is bounded and weakly Haar measurable, we conclude $x^{*} \circ(\Psi-\varphi) * f=c\left(x^{*}, f\right)$, where $c\left(x^{*}, f\right) \in \mathbf{C}$ for each $x^{*} \in X^{*}$ and $f \in C(G)$. Since $x^{*} \circ(\Psi-\varphi)$ is integrable, there is $\left(f_{n}\right) \subset C(G)$ such that $x^{*} \circ(\Psi-\varphi) * f_{n}(t) \rightarrow x^{*} \circ(\Psi-\varphi)(t)$ almost everywhere on $G$. This implies that $x^{*} \circ(\Psi-\varphi)(t)=c\left(x^{*}\right)$ almost everywhere. Since the dual of $X$ has a countable separating set $M, \Psi(t)=\varphi(t)+a$ with $a \in X$ almost everywhere. This proves that $\varphi$ is continuous.

Special $X$ where Corollary 2.3 can be applied are the spaces of test functions or Schwartz distributions; $X$ separable Fréchet space ; $X$ Fréchet space and $\varphi$ is strongly measurable (meaning $j \circ \varphi$ is Bochner measurable for each $j$ corresponding to a countable number of seminorms determining the topology of $X$ ).

Example 2.4. (i) Consider the compact multiplicative group $T=\left\{e^{i t}: 0 \leq t<\right.$ $2 \pi\}$. The function $\varphi\left(e^{i t}\right)=0$ if $t \in Q, \varphi\left(e^{i t}\right)=1$ if $t \in \mathbf{R}-Q$, shows that under the assumptions of Corollary 2.3 (i), $\varphi$ itself is not necessarily continuous and the continuous extension of $\left.\varphi\right|_{\Gamma}$ is almost everywhere equal to $\varphi-1$.

(ii) Let $X=l^{2}(T)$, the Hilbert space of all square summable real valued functions defined on the compact group $T$. Denote by $\chi_{t}$ the characteristic function of the point set $\{t\}$ of $T$. Define $\psi: G \rightarrow l^{2}(T)$ by $\psi(t)=\chi_{t}$. Obviously, $x^{*} \circ \psi$ is a bounded measurable almost everywhere zero function for each $x^{*} \in\left(l^{2}(T)\right)^{*}$, but $\psi \neq 0$ almost everywhere. This demonstrates that for our proof the condition of Corollary 2.3 (ii) that the dual of $X$ has a countable separating subset is necessary.

(iii) There exists a non-measurable function (see [13, p. 87]) $\chi: \mathbf{R} \rightarrow \mathbf{C}$ such $\chi(x+y)=\chi(x) \chi(y),|\chi(x)|=1$ for all $x, y \in \mathbf{R}$ and $\chi(x)=1$ for all $x \in Q$. We have $\Delta_{r} \chi(x)=0$ for all $r \in Q$ and $x \in \mathbf{R}$. This means that measurability is necessary in Corollary 2.3 (ii).

We note that Theorem 2.1 extends [2, Theorem 2.1] to a more general setting. Corollary 2.3 strengthens recent results in [16, Theorem 1, Lemma 1].

\section{Properties of D-type Groups}

In this section we study D-type groups and give the relationship between weak Namioka groups and those of weak $\Delta$-type. 
Proposition 3.1. (i) If $G$ is a semigroup with identity e, then $G$ is of D-type if and only if there is a sequence of open neighbourhoods $\left(W_{n}\right)$ of e such that

$$
\begin{aligned}
& \text { for any sequences } n_{k}, t_{k} \text { with } n_{k}<n_{k+1}, t_{k} \in W_{n_{k}} \text { for } \\
& k \in \mathbf{N},\left(\tau_{m}\right) \text { defined by } \tau_{m}:=t_{m} t_{m-1} \ldots t_{1} \text { for } m \in \mathbf{N}, \\
& \text { has a convergent subnet. }
\end{aligned}
$$

(ii) If $G$ is a semigroup with identity $e$, if $\left(V_{n}\right)$ is a sequence of neighbourhoods of e such that $V_{n} \subset W_{n}$ for all $n \in \mathbf{N}$ and if $\left(W_{n}\right)$ satisfies (D), then $\left(V_{n}\right)$ also satisfies (D).

(iii) If $G$ is a quasitopological group which either (a) satisfies the first axiom of countability and is (sequentially) complete, or (b) is locally countably compact, then $G$ is of D-type.

Proof. (i) 'if' is obvious. 'only if': First, choose open neighbourhood $U_{n}$ with $e \in U_{n} \subset W_{n}$; then $V_{n}:=\bigcap_{k=1}^{n} U_{k}$ are open neighbourhoods of $e$ with $V_{n+1} \subset$ $V_{n} \subset W_{n}$. If $t_{k} \in V_{n_{k}}$, then $t_{k} \in V_{k} \subset W_{k}$ since $k \leq n_{k}$ for all $k \in \mathbf{N}$. By (D), $\tau_{m}$ has a convergent subnet.

(ii) Direct verification.

(iii) Choose $\left(V_{n}\right)$ such that

$$
\begin{aligned}
& \text { is an open neighbourhood of } e \text { and } V_{n+1} V_{n+1} \subset V_{n} \text { for } \\
& n \in \mathbf{N} \text {. }
\end{aligned}
$$

This is possible since $G$ is a quasitopological group. If $G$ satisfies (a), and $\left(W_{n}\right)$ is a neighbourhood basis of $e$, choose additionally the $V_{n}$ as subsets of $W_{n}$, and if $G$ satisfies (b), choose $V_{1}$ countably compact. Then $\left(V_{n}\right)$ satisfies (D).

In the following if $A \subset G$, then $\bar{A}$ will denote the closure of $A$ and $\bar{A}^{o}$ the interior of $\bar{A}$.

Theorem 3.2. If $G$ is a quasitopological group of D-type, then

(i) $G$ is a Baire space if additionally $G$ is regular.

(ii) $G$ is $\sigma$-complete; i.e Cauchy sequences are convergent.

Proof. (i) By contradiction: Assume that $U$ is a non-empty open subset of $G$ and $U=\bigcup_{n=1}^{\infty} E_{n}$ with ${\overline{E_{n}}}^{\circ} \cap U=\emptyset$ for $n \in \mathbf{N}$. One has $U=\bigcup_{n=1}^{\infty} F_{n} \cap U$ with $F_{n}={\overline{E_{n}}}-{\overline{E_{n}}}^{\circ}$ closed in $G, F_{n}^{o}=\emptyset$. Since for closed sets $A, B$ with empty interior also $A \cup B$ has empty interior, one can assume $F_{n} \subset F_{n+1}$. Since for $M$ open respectively closed subset of $G$ also $t M$ and $M t$ are open respectively closed subsets, one can further assume $e \in U$. By Proposition 3.1 and regularity of $G$, we can choose $\left(V_{n}\right)$ satisfying (3.1) and $V_{n} V_{n} \subset V_{n-1} \subset \overline{V_{1} V_{1}} \subset U, n>1$. Inductively construct sequences $\left(M_{n}\right),\left(t_{n}\right),\left(U_{n}\right),\left(S_{n}\right),\left(W_{n}\right)$ with : $M_{1}=F_{1}, t_{1} \in V_{1}-M_{1}, U_{1}$ and $S_{1}$ open with $e \in U_{1} \subset V_{1}$ and $M_{1} \subset S_{1}, S_{1} \cap U_{1} t_{1}=\emptyset, W_{1}$ open with $e \in W_{1}$, $W_{1} W_{1} \subset U_{1}$. If $n>1$ and $M_{j}, \ldots, W_{j}$ are constructed for all $1 \leq j \leq n-1$, then $M_{n}:=F_{n} t_{1}^{-1} \ldots t_{n-1}^{-1}, t_{n} \in W_{n-1} \cap V_{n}-M_{n}, U_{n}, S_{n}, W_{n}$ open with $e \in U_{n} \subset W_{n-1}$, $M_{n} \subset S_{n}, U_{n} t_{n} \cap S_{n}=\emptyset, W_{n} W_{n} \subset U_{n}$. Such $t_{n}, \ldots, W_{n}$ exist, since by the above $M_{n}$ is closed with empty interior and $G$ is a regular topological semigroup. If $\tau_{m}:=t_{m} t_{m-1} \ldots t_{1}=t_{m} t_{m-1} \ldots t_{k+1} \tau_{k}, 1 \leq k<m$, then $t_{m} \ldots t_{k+1} \in U_{n_{k}}$ (proof by induction on $p, m=k+p$, since $\left.W_{n} \subset U_{n} \subset W_{n-1} \subset U_{n-1}, t_{k+1} \in W_{k}\right)$. Thus if $1 \leq k<m$,

$$
\tau_{m} \in U_{k} \tau_{k}, U_{k} \tau_{k} \text { disjoint with } S_{k} t_{k-1} \ldots t_{1} \supset F_{k} .
$$


By Proposition 3.1 (i) there is a subnet $\left(\tau_{m(i)}\right)$ converging to some $\tau \in G$; since $\tau_{m} \in U_{1} \tau_{1} \subset V_{1} t_{1} \subset V_{1} V_{1} \subset \overline{V_{1} V_{1}} \subset U, \tau \in U$. Thus there is $n_{0}$ with $\tau_{n_{0}} \in F_{n_{0}}$; furthermore there is $i_{0}$ with $m(i)>n_{0}$ if $i \geq i_{0}$. Since $\tau$ belongs to the open subset $S_{n_{0}} t_{n_{0}-1} \ldots t_{1}$, there is $i \geq i_{0}$ with $\tau_{m(i)} \in S_{n_{0}} t_{n_{0}-1} \ldots t_{1}$ and $m(i)>n_{0}$, contradicting (3.3). This proves (i).

(ii) Let $\left(V_{n}\right)$ be a sequence of open sets with (D). Let $\left(a_{n}\right)$ be a Cauchy sequence of $G$, that is $a_{n} a_{m}^{-1} \rightarrow e$ as $n, m \rightarrow \infty$. One chooses inductively $n_{k}<n_{k+1}$ such that $a_{n} a_{m}^{-1} \in V_{k}$ for $n, m \geq n_{k}$. Indeed, choose $n_{1} \in \mathbf{N}$ such that $a_{n} a_{m}^{-1} \in V_{1}$ for $n, m \geq n_{1}$. Choose $n_{2}$ such that $a_{n} a_{m}^{-1} \in V_{2}$ for $n, m \geq n_{2}>n_{1}$. We can proceed by induction. If $t_{k}=a_{n_{k+1}} a_{n_{k}}^{-1}$, then $t_{k} \in V_{k}$ for $k \in \mathbf{N}$ and $\tau_{m}=t_{m} t_{m-1} \ldots t_{1}=$ $a_{n_{m}} a_{n_{1}}^{-1}$. It follows by Proposition 3.1 (i), the sequence $\left(a_{n_{k}}\right)$ has a convergent subnet. Hence $\left(a_{n}\right)$ is convergent for $\left(a_{n}\right)$ is Cauchy.

Corollary 3.3. Let $G$ be a quasitopological group satisfying the first axiom of countability. Then $G$ is of D-type if and only if it is complete.

Proof. For sufficiency, choose a basis system of neighbourhoods $\left(V_{n}\right)$ of $e$ satisfying (3.2). Let $\left(t_{k}\right) \subset G, t_{k} \in V_{n_{k}}$ and $n_{k}<n_{k+1}$. Let $\tau_{m}=t_{m} t_{m-1} \ldots t_{1}$. Then $\left(\tau_{m}\right)$ is a Cauchy sequence from $G$ and therefore it is convergent, for $G$ is complete. Hence $\left(V_{n}\right)$ satisfies (D), by Proposition 3.1 (i). Necessity follows from Theorem 3.2.

Proposition 3.4. If $G$ is a weak Namioka semitopological group then $G$ is of weak $\Delta$-type.

Proof. First, assume that $X$ is a real normed space. Let $Y=B^{*}$ with $B^{*}$ the unit ball of the dual Banach space $X^{*}$ of $X$. Let $\Phi: G \times B^{*} \rightarrow \mathbf{R}$ be defined by $\Phi\left(t, x^{*}\right)=x^{*} \circ \varphi(t)$. If $\varphi$ is as assumed in the definition of weak $\Delta$-type, then by Corollary 2.2 (i) $x^{*} \circ \varphi$ is continuous on $G$. Since $x^{*} \circ \varphi(t)$ is continuous for each fixed $t \in G$, it follows that $\Phi\left(t, x^{*}\right)$ is separately continuous on $G \times B^{*}$. As $G$ is a weak Namioka space, $\Phi$ is continuous on $\{a\} \times B^{*}$ with $a \in G$. Using the compactness of $B^{*}, \varphi$ is continuous at $a$. By the identity $\varphi(t h)=\Delta_{h} \varphi(t)-\Delta_{a} \varphi(t)+\varphi(t a), a, h, t \in$ $G, \varphi$ is continuous on $G$. Now, let $X$ be locally convex space. Let $p$ be a seminorm of $X$. The above argument can be applied to $j \circ \varphi$. This proves that $\varphi$ is continuous on $G$ and implies that $G$ is of weak $\Delta$-type.

The class of Namioka spaces contains all separable Baire spaces [6], [15] and all strongly countably complete regular spaces (see [8], [14]). The latter spaces are contained in the class of $\sigma$-well $\alpha$-favorable spaces defined in [8]. This implies that semitopological groups of weak $\Delta$-type include all Namioka semitopological groups; in particular all separable Baire semitopological groups, all strongly countably complete regular semitopological groups, all $\sigma$-well $\alpha$-favorable and all $\sigma-\beta$ defavorable semitopological groups (see [15]).

Example 3.5. (i) Let $J$ be a linear rational basis of $\mathbf{R}$ (see [13, Satz 3]) and $\xi \in J$. Let $G$ be the subgroup of $\mathbf{R}$ consisting of all rational linear combinations with elements from $J \backslash\{\xi\}$. Then $G$ is a metric topological group, it is Baire separable and thus a Namioka space, thus of a weak $\Delta$-type. Since $G$ is not sequentially complete it is not of $D$-type, by Theorem 3.2 (ii).

(ii) Denote by $\mathbf{R}_{s}$ the additive group of reals endowed with the topology generated by the basis neighbourhood system $\left\{\left[0, x[: x>0\}\right.\right.$ of 0 . Then $\mathbf{R}_{s}$ is a normal quasitopological group which is not a topological group. It is of weak $\Delta$-type but not of $D$-type. 
Problem 1. Is every semitopological group of weak $\Delta$-type a weak Namioka space?

Problem 2. Does the class of semitopological groups of weak $\Delta$-type properly contain $\Delta$-type semitopological groups?

\section{Differences on D-type topological Groups}

In this section, we study the relation between groups of D-type and those of $\Delta$-type. Also, we discuss differences on weak Namioka semitopological groups and underline some possible extensions to the case where $X$ is a separable linear metric space with separating dual space and the case where $G$ is an Abelian group endowed with two topologies.

Theorem 4.1. Let $G$ be a D-type regular quasitopological group and $X$ be a locally convex space. Assume that the function $\varphi: G \rightarrow X$ is bounded on an open set $U$, $e \in U$ and its difference $\Delta_{h} \varphi$ is continuous at e for all $h \in U$. Then $\varphi$ is continuous at e. In short, a D-type regular quasitopological group is of $\Delta$-type.

Proof. First we give the proof in the case where $X$ is a normed space. Let $x^{*}$ be an element of the dual Banach space $X^{*}$. Consider $x^{*} \circ \varphi$. By Corollary 2.2 (i), $x^{*} \circ \varphi$ is continuous on $U$. This means that

$\varphi$ is weakly continuous on $U$.

Since $G$ is a regular quasitopological group, by Proposition 3.1 (i), (iii) we can select $V_{0}$ and $\left(V_{n}\right)$ all open neighbourhoods of $e$ satisfying (3.1) and $\overline{V_{n} V_{n}} \subset V_{n-1} \subset U$. We also can assume that $X$ is a Banach space and that $\varphi(e)=0$. Assuming that $\varphi$ is not continuous at $e$ there exists $\epsilon_{o}>0$ and a sequence $\left\{t_{n}\right\}$ satisfying

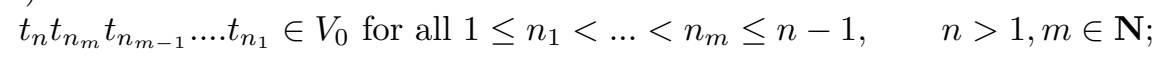

$$
\begin{aligned}
\left\|\varphi\left(t_{n} t_{n_{m}} t_{n_{m-1}} \ldots t_{n_{1}}\right)-\varphi\left(t_{n}\right)-\varphi\left(t_{n_{m}} t_{n_{m-1}} \ldots t_{n_{1}}\right)\right\|<\epsilon_{o} / 2^{n} \\
\text { for all } 1 \leq n_{1}<\ldots<n_{m} \leq n-1, n>1, m \in \mathbf{N} .
\end{aligned}
$$

Indeed, by our assumptions we can find $\epsilon_{o}>0$ and $t_{1} \in V_{1}$ such that $\left\|\varphi\left(t_{1}\right)\right\| \geq \epsilon_{o}$. Put $E_{\tau}(\epsilon)=\left\{t:\left\|\Delta_{\tau} \varphi(t)-\Delta_{\tau} \varphi(e)\right\|<\epsilon\right\}$. From the continuity of $\Delta_{t_{1}} \varphi$ at $e$ it follows that the set $E_{t_{1}}\left(\epsilon_{o} / 2\right) \cap V_{1} t_{1}^{-1} \cap V_{2} \subset U$ is a neighbourhood of $e$. Hence there exists $t_{2} \in E_{t_{1}}\left(\epsilon_{o} / 2\right) \cap V_{1} t_{1}^{-1} \cap V_{2}$ such that $\left\|\varphi\left(t_{2}\right)\right\| \geq \epsilon_{o}$. The construction can proceed by induction. If $t_{1}, t_{2} \ldots, t_{n-1}$ are found, then $t_{n}$ can be selected such that $\left\|\varphi\left(t_{n}\right)\right\| \geq \epsilon_{o}$ and $t_{n} \in E_{t_{n_{m}} t_{n_{m-1}} \ldots t_{n_{1}}}\left(\epsilon_{o} / 2^{n}\right) \cap V_{1} t_{n_{1}}^{-1} \ldots . t_{n_{m}}^{-1} \cap V_{n}$ for all $1 \leq$ $n_{1}<\ldots<n_{m} \leq n-1, n>1, m \in \mathbf{N}$. Clearly, (4.2)-(4.4) are satisfied. By Proposition 3.1 (i), for any subsequence $\pi=\left(t_{n_{m}}\right)$ with $n_{k}<n_{k+1}$ the sequence $\left(\tau_{m}\right)$ defined by $\tau_{m}=t_{n_{m}} t_{n_{m-1}} \ldots . t_{n_{1}}$ has a subnet, say $(\tau)_{i \in \Lambda}$ which converges to $\tau_{\pi} \in G$. It follows from the choice of $\left(V_{n}\right)$ that $\tau \in U$. Set $s_{m}=\sum_{k=1}^{m} \varphi\left(t_{n_{k}}\right)$ and $\tilde{s}_{m}=\varphi\left(t_{n_{m}} t_{n_{m-1} \ldots .} t_{n_{1}}\right)$. Using (4.1), we get

$$
(\tilde{s})_{i \in \Lambda} \text { weakly converges to } \varphi\left(\tau_{\pi}\right) \text {. }
$$

Let $\sigma_{m}=s_{m}-\tilde{s}_{m}$. By $\sigma_{m}-\sigma_{m+p}=\varphi\left(t_{n_{m+p}} \ldots . t_{n_{1}}\right)-\varphi\left(t_{n_{m+p}}\right)-\varphi\left(t_{n_{m+p-1}} \ldots . t_{n_{1}}\right)+$ $\ldots .+\varphi\left(t_{n_{m+1}} \ldots . t_{n_{1}}\right)-\varphi\left(t_{n_{m+1}}\right)-\varphi\left(t_{n_{m}} \ldots t_{n_{1}}\right)$, and (4.4), we conclude that $\left(\sigma_{m}\right)$ is a Cauchy sequence. Denote its limit by $y_{\pi}$. Arguing as in the proof of (2.5), we get $\left(s_{m}\right)$ and $(\tilde{s})_{m}$ are weakly sequentially Cauchy. From (4.5), we conclude 
$\sum_{k=1}^{\infty} \varphi\left(t_{n_{k}}\right)=y_{\pi}+\varphi(\tau)$ weakly. By Orlicz's Theorem, it follows that $\sum_{n=1}^{\infty} \varphi\left(t_{n}\right)$ is convergent, contradicting (4.2).

Now, let $X$ be locally convex space. Let $p$ be a continuous seminorm of $X$. The above arguments can be applied to $j \circ \varphi$. This proves that $\varphi$ is continuous at $e$.

Remark 4.2. (i) Theorem 4.1 holds true for separable linear metric spaces $X$ with separating dual space. In this case we apply a Theorem of Kalton [12, corollary of Theorem 3] which extends Orlicz's Theorem.

(ii) Theorem 4.1 holds correspondingly if only $\varphi: U \rightarrow X$ is given, $U$ neighbourhood of $e$ (with $V V \subset U,\left.\Delta_{h} \varphi\right|_{V}$ continuous at $e$ ).

Also, using [12, Theorems 3 and 7] and the same method of the proof of Theorem 4.1, one can obtain:

Theorem 4.3. Let $G$ be a D-type regular quasitopological group and $X$ an Abelian group endowed with two Hausdorff topologies $\rho$ and $\tau$ such that $\rho \leq \tau,(X, \tau)$ is a separable topological group and one of the following two conditions is satisfied:

$\left(K_{1}\right)(X, \rho)$ is separable and $\tau$ is complete and metrizable;

$\left(K_{2}\right) \tau$ has a base of $\rho$-closed neighbourhoods of 0.

Let $\varphi: G \rightarrow X$ be $\rho$-continuous on an open $U$ with $e \in U$ and for each $h \in U$ the difference $\Delta_{h} \varphi$ is $\tau$-continuous at $e$. Then $\varphi$ is $\tau$-continuous on $U$.

\section{UNIFORMLY CONTINUOUS DIFFERENCES}

Let $G$ be a semitopological group and $X$ a Hausdorff topological Abelian group. Denote by $C_{r u}(G, X)$ the space of all right uniformly continuous functions (see section 1) endowed with the topology of uniform convergence on $G$. If $X$ is locally convex, $C_{r u b}(G, X)$ will stand for the subspace of bounded functions of $C_{r u}(G, X)$.

In this section $\varphi: G \rightarrow X$ will denote a function satisfying the following:

$$
\begin{aligned}
& \Delta_{h} \varphi \in C_{r u}(G, X) \text { for all } h \in U \text {, where } U \text { is some neigh- } \\
& \text { bourhood of } e \text {. }
\end{aligned}
$$

Let $\psi, \tilde{\psi}$ be defined respectively by $\psi(h)=\Delta_{h} \varphi$ for $h \in G$ and $\tilde{\psi}(h)=\Delta_{h} \varphi$ for $h \in U$ and $\tilde{\psi}(h)=0$ for all $h \in G \backslash U$. We give the following

Lemma 5.1. Let $\varphi: G \rightarrow X$ and $\psi, \tilde{\psi}$ be as above. Then

(i) $\varphi \in C_{r u}(G, X)$ if and only if (5.1) holds and $\psi_{U}: U \rightarrow C_{r u}(G, X)$ is continuous at e.

(ii) (5.1) implies $\Delta_{k} \psi \in C_{r u}\left(G, C_{r u}(G, X)\right)$ for all $k \in U$.

(iii) (5.1) implies $\Delta_{k} \tilde{\psi}$ is continuous at e for all $k \in V$, where $V$ is a neighbourhood of e satisfying $V V \subset U$.

Proof. (i) If $\varphi \in C_{r u}(G, X)$, not only left, but also right translates $R_{h} \varphi(t):=\varphi(t h)$ are right uniformly continuous on $G\left(h^{-1} s h\right.$ is continuous at $\left.s=e\right)$, so (5.1) holds for $U=G ; \Delta_{v} \psi(e)=\Delta_{v} \varphi$ gives (i).

(ii) follows from $\Delta_{k} \psi(h)=\Delta_{h k} \varphi-\Delta_{h} \varphi=R_{h} \Delta_{k} \varphi$ if $h, k \in G$, and the proof of

(iii) follows from $\Delta_{k} \tilde{\psi}(h)=\Delta_{h k} \varphi-\Delta_{h} \varphi=R_{h} \Delta_{k} \varphi$ if $h, k \in V$.

Theorem 5.2. Let $G$ be a $\Delta$-type quasitopological group, $X$ locally convex and $\varphi: G \rightarrow X$. If $\varphi$ satisfies (5.1) and if $\tilde{\psi}$, defined as above, is bounded in $C_{r u}(G, X)$ on some neighbourhood $\tilde{U}$ of e, then $\varphi$ is right uniformly continuous. 
Proof. Choose a neighbourhood $V$ of $e$ such that $V V \subset U \cap \tilde{U}$. By assumption on $\left.\tilde{\psi}\right|_{\tilde{U}}$, one has now $\tilde{\psi}: G \rightarrow C_{r u b}(G, X)$, and $\left.\tilde{\psi}\right|_{V}$ is bounded in $C_{r u b}(G, X)$. By Lemma 5.1 (iii), $\Delta_{k} \tilde{\psi}: G \rightarrow C_{r u}(G, X)$ is continuous at $e$ for each fixed $k \in V$, this also as a function from $G$ to $C_{r u b}(G, X)$. Since $C_{r u b}(G, X)$ is locally convex, by definition of $\Delta$-type, $\tilde{\psi}$ is continuous at $e$, this also as a function: $V \rightarrow C_{r u}(G, X)$. Therefore, $\varphi$ is right uniformly continuous, by Lemma 5.1.

Theorem 5.3. Let $G$ be a weak Namioka semitopological group, $X$ locally convex. If $\varphi: G \rightarrow X$ is bounded and satisfies (5.1) with $U=G$, then $\varphi$ is right uniformly continuous.

Proof. By Proposition 3.4, $G$ is of weak $\Delta$-type. The assumptions imply that $\psi$, $\psi(v):=\Delta_{v} \varphi$ is bounded and by Lemma $5.1, \Delta_{k} \psi \in C_{r u b}\left(G, C_{r u b}(G, X)\right)$ for all $k \in G$. By Definition 1.1 (ii), $\psi$ is continuous at $e$. Therefore, $\varphi$ is right uniformly continuous, by Lemma 5.1.

Remark 5.4. (i) (5.1) is necessary in Theorem 5.2 by Lemma 5.1; for normed $X, \psi$ bounded on some neighbourhood is necessary.

(ii) Theorem 5.2 is also true if $X$ is a separable metric linear space with separating dual and $G$ additionally is separable. (For Remark 4.2, here $C_{r u d}(G, X)$ has to be separable metric, thus $G$ separable; the dual of $C_{r u d}(G, X)$ is then automatically separating; $C_{r u d}(G, X)$ is the set of all $\varphi \in C_{r u}(G, X)$ with $d(\varphi, 0)<\infty, d(\varphi, \psi)=$ $\sup _{t \in G} d_{X}(\varphi(t), \psi(t))$.

The assumptions of uniform boundedness of the $\Delta_{h} \varphi$ in Theorem 5.2 can be weakened as follows

Corollary 5.5. Let $G$ be a D-type regular quasitopological group, $X$ locally convex, $\Delta_{h} \varphi$ right uniformly continuous on $G$ for each $h \in G, \Delta_{h} \varphi$ bounded on $G$ for each fixed $h$ from some nonempty open set $V$, and $\varphi$ bounded on some other nonempty open set $W$. Then $\varphi$ is right uniformly continuous on $G$.

Proof. By left translation one can assume $\varphi$ is bounded on some neighbourhood of $e$. As in the proof of Theorem 4.1, one can assume $X$ is normed. If $\Delta_{h} \varphi$ is bounded for $h \in V$, it is for $h \in U:=V V^{-1}$, a neighbourhood of $e$. By Theorem 4.1, $\varphi$ is continuous at $e$, then on $G$. If $M_{n}:=\left\{u \in U:\left\|\Delta_{u} \varphi(s)\right\| \leq n\right.$, for all $\left.s \in G\right\}$, then $M_{n}$ are closed in $U$ and $U=\bigcup_{n=1}^{\infty} M_{n}$. By Theorem 3.2 (i), some $M_{n}$ contains a nonempty open $P$; then $\left\|\Delta_{u} \varphi(s)\right\| \leq 2 n$ for all $s \in G, u \in P P^{-1}$, = neighbourhood of $e$ and Theorem 5.2 can be applied.

Remark 5.6. (i) None of the conditions of Theorem 5.2 can be omitted. All the differences of the function $\varphi: \mathbf{R} \rightarrow c_{o} ; \varphi(t)=(\sin (t / n))$ are almost periodic. This means that $\Delta_{h} \varphi$ is bounded and uniformly continuous on $\mathbf{R}$ for $h \in \mathbf{R}$ endowed with the Bohr topology, but $\varphi$ is not continuous at any point of $\mathbf{R}$ with the Bohr topology (see [1, p. 54]). This also gives an example of a totally bounded topological group which is not of D-type or of $\Delta$-type.

(ii) For continuous $\varphi$ and complete metric $G$, Corollary 5.5 is also true if $\Delta_{h} \varphi \in$ $C_{r u}(G, X)$ for $h \in P$ where $P$ is second category in $G$ and $V$ is also only of second category.

(iii) If $G$ is locally compact, ' $V$ and $W$ of positive Haar measure' also suffices in Corollary 4.4 ([9] proof of Theorem 2.3 there; $V^{-1} V$ is a neighbourhood of $e$ ). 
(iv) With $G$ obviously also $G \times K$ is of D-type if $K$ is countably compact; this gives examples of topological groups where Theorem 5.2 and Corollary 5.5 hold, but the groups are neither locally compact nor metrizable.

(v) Because of Proposition 3.1, the above results subsume earlier results of [11, Lemma 4, p. 266] and [9, section II.4, corollary] for locally compact abelian groups. Theorem 5.3 also subsumes [3, Theorem 4.1].

(vi) The assumption $\psi \mid U$ bounded in $C_{r u}(G, X)$ is not necessary, similarly for the weaker assumption in Corollary 5.5. We give the following example.

Let $G=\mathbb{Z}^{\mathbf{N}}$, the topological product of countable many copies of the integers $\mathbb{Z}$ with their discrete topologies. Let $X=\mathbb{R}^{\mathbf{N}}$ with the topology of pointwise convergence. If $\varphi\left((z)_{n}\right):=\left(z_{n}^{2}\right)_{n \in \mathbf{N}}$, then $\varphi$ is uniformly continuous, but no $\Delta_{h} \varphi, h \neq e$ is bounded. So, $\Delta_{h} \varphi$ only belong to $C_{r u}(G, X)$ but do not belong to $C_{r u b}(G, X)$. So condition (5.1) is more applicable as it stands.

The function $\varphi(t)=t^{2}, G=X=\mathbf{R}$ shows that the uniform continuity of $\Delta_{h} \varphi$ for all $h \in \mathbf{R}$ alone is not enough for uniform continuity of $\varphi$.

(vii) The boundedness of $\varphi$ in Theorem 5.3 can be replaced by: To each continuous seminorm $p$ of $X$ there exists an open $V=V(p)$ with $e \in V$ and $\sup \left\{p\left(\Delta_{h} \varphi(t)\right)\right.$ : $h \in V, t \in G\}<\infty$. This condition is also necessary. If $G$ is a Baire space, the boundedness on $G$ only of $\Delta_{h} \varphi$ for each fixed $h$ from some open $V_{0}$ (and $\varphi$ bounded on some open $W$ ) would be enough as in Corollary 5.5. Similarly Theorem 5.2 and other results of this section can be strengthened.

\section{REFERENCES}

[1] L. Amerio and G. Prouse, Almost Periodic Functions and Functional Equations, VanNostrand Reinhold Company, 1971. MR 48:419

[2] B. Basit and M. Emam, Differences of functions in locally convex spaces and applications to almost periodic and almost automorphic functions, Annales Polonici Math. 41 (1983), 193-201. MR 85d:43005

[3] B. Basit and A. J. Pryde, Differences of vector-valued functions on topological groups, Proc. Amer. Math. Soc. 124 (1996), 1969-1975. CMP 95:08

[4] J. F. Berglund, H. D. Junghenn and P. Milnes, Analysis on Semigroups: Function Spaces, Compactification, Representations, Canadian Mathematical Society Series of Monographs, A Wiley-Interscience Publication, 1989. MR 91b:43001

[5] C. Bessaga and A. Pelczynski, On bases and unconditional convergence of series in Banach spaces, Studia Math. 17 (1958), 151-164. MR 22:5872

[6] J. Calbrix et J. P. Troallic, Application séparément continues, C.R.Acad.Sci. Paris, série A-B, 288 (1979), 647-648. MR 80c:54009

[7] G. Choquet, Lectures on Analysis, Vol. 1, Benjamin, New York and Amesterdam, 1969. MR 40:3252

[8] J.P.R Christensen, Joint continuity of separately continuous functions, Proc. Amer. Math. Soc. 82 (1981), 455-461. MR 82h:54012

[9] C. Datry and G. Muraz, Analyse harmonique dans les modules de Banach I : propriétés générales, Bull. Science Mathematique 119 (1995), 299-337. CMP 95:15

[10] F. Galvin, G. Muraz et P. Szeptycki, Fonctions aux différences $f(x)-f(a+x)$ continues, C.R.Acad.Sci. Paris, série I, 315 (1992), 397-400. MR 94b:39035

[11] H. Günzler, Integration of almost periodic functions, Math. Zeit. 102, (1967), 253- 287. MR 36:3066

[12] N. J. Kalton, Subseries convergence in topological groups and vector spaces, Israel J. Math. 10 (1970), 402-412. MR 45:3628

[13] W. Maak, Fastperiodische Funktionen, Springer-Verlag, 1967. MR 35:5860

[14] I. Namioka, Separate continuity and joint continuity, Pacific Journal of Math. 51 (1974), 515-531. MR 51:6693 
[15] J. Saint-Raymond, Jeux topologiques et espaces de Namioka, Proc. Amer. Math. Soc. 87 (1983), 499-504. MR 83m:54060

[16] F. Watbled, Ensemble de Rosenthal pour des Fonctions a Valeurs Banach, C.R.Acad.Sci. Paris, série I, 318 (1994), 333-336. MR 94m: 43007

Department of Mathematics, Monash University, Clayton Vic. 3168, Australia

E-mail address: bbasit@vaxc.cc.monash.edu.au

Mathematisches Seminar der Universität Kiel, Ludewig-Meyn-Str., 424098 Kiel, DEUTSCHLAND

E-mail address: guenzler@math.uni-kiel.de 